\title{
On the Lambda-evolution of galaxy clusters
}

\author{
V. G. Gurzadyan ${ }^{1,2, a}$, A. A. Kocharyan ${ }^{3}$, A. Stepanian ${ }^{1}$ \\ ${ }^{1}$ Center for Cosmology and Astrophysics, Alikhanian National Laboratory, Yerevan State University, Yerevan, Armenia \\ 2 SIA, Sapienza Universita di Roma, Rome, Italy \\ ${ }^{3}$ School of Physics and Astronomy, Monash University, Clayton, VIC, Australia
}

Received: 14 November 2019 / Accepted: 26 December 2019 / Published online: 11 January 2020

(C) The Author(s) 2020

\begin{abstract}
The evolution of galaxy clusters can be affected by the repulsion described by the cosmological constant. This conclusion is reached within the modified weak-field General Relativity approach where the cosmological constant $\Lambda$ enables to describe the common nature of the dark matter and the dark energy. Geometrical methods of theory of dynamical systems and the Ricci curvature criterion are used to reveal the difference in the instability properties of galaxy clusters which determine their evolutionary paths. Namely, it is shown that the clusters determined by gravity with $\Lambda$ repulsion tend to become even more unstable than those powered only by Newtonian gravity, the effect to be felt at cosmological time scales.
\end{abstract}

\section{Introduction}

The nature of the dark sector - dark matter and dark energy - remains a major puzzle for fundamental physics in spite of intense observational, experimental and theoretical investigations of the last decades. The recently sharpened $H$ tension, i.e. the discrepancy between the Hubble constant determinations from Planck's data and observations at lower redshift [1,2], activated the discussions regarding beyond $\Lambda \mathrm{CDM}$ and new physics $[3,4]$.

The modified weak-field General Relativity (GR) provides one of recent approaches to describing the dark sector [57]. That modification is based on Newton's theorem on the identity of gravity of a sphere and of a point mass situated in its center and enables one to conclude to the common nature of the dark matter and dark energy, both being described by the cosmological constant [6,7]. That approach also offers a solution to the $H$-tension [8]. The cosmological constant $\Lambda$ within that approach acts as a fundamental constant along with the gravitational constant $G$ [9], with consequences also for conformal cyclic cosmology [10]. The non-particle nature of the dark matter is concluded to in [11].

If the gravitational interaction at galaxy cluster scales is defined not only by an attracting force but also by a repulsive force due to the cosmological constant, then the latter can influence the evolution of galaxy clusters. Analogous modified gravity effects are among the discussed ones with respect to various astrophysical systems, from celestial mechanical scales [12] to those of cosmological structure formation [13], whereas the dedicated experimental tests of General Relativity (e.g. $[14,15])$ still do not reach those effects. Other testing opportunities are provided by the lensing of galaxies $[16,17]$.

Here we will try to reveal a possible difference in the dynamical evolution of two types of galaxy clusters, those determined by usual Newtonian gravity (standard $N$-body problem) and those by modified gravity with the repulsion term. We use geometrical methods of the theory of dynamical systems $[18,19]$ first applied to gravitational $N$-body dynamics in [20] to describe their chaos and relaxation; for further application of those methods in General Relativity see $[21,22]$. The Ricci curvature criterion of relative instability, which we use here, was introduced in [23] and has been applied to different types of gravitational systems (e.g. [24]). Our aim is not the study of entire evolution of galaxy clusters affected by modified gravity, which will need an extensive strategy of $N$-body simulations but to verify if the $\Lambda$ term is able to influence the cluster dynamics and the evolution. Such an approach appears informative for nonlinear systems, as known since the renowned Fermi-Pasta-Ulam study [25].

The results of our analysis indicate that $\Lambda$-gravity does affect the instability features of galaxy clusters. We note that previously it was shown that the cosmological constant is able to introduce a time arrow for the system [26].

\footnotetext{
a e-mail: gurzadyan@yerphi.am
} 


\section{Newton's theorem and $\Lambda$}

Proceeding from Newton's theorem on the "sphere-point" identity and the resulting weak-field modification of General Relativity, one arrives at the metric [6] $(c=1)$

$g_{00}=1-\frac{2 G m}{r}-\frac{\Lambda r^{2}}{3} ; \quad g_{r r}=\left(1-\frac{2 G m}{r}-\frac{\Lambda r^{2}}{3}\right)^{-1}$.

The general functions for force and potential i.e. $U(r)$ and $\mathbf{F}(r)$ satisfying Newton's theorem used for the above weakfield limit of GR have the form (for derivation and discussion see $[5,6,27])$

$U(r)=-\frac{A}{r}-\frac{B}{2} r^{2} ; \quad \mathbf{F}(r)=-\nabla_{r} U(r)=\left(-\frac{A}{r^{2}}+B r\right) \hat{\mathbf{r}}$.

Here the second term leads to the cosmological term in the solutions of Einstein equations, so that the cosmological constant $\Lambda$ enters also the weak-field GR regimes, e.g. in the Hamiltonian dynamics of galaxy clusters [8].

Crucial feature of the force law of Eq. (2) is that it defines non-force-free field inside a spherical shell, contrary to Newton's gravity law when the shell has no influence in its interior. In this regard we mention the observational indications that the galactic halos do determine the properties of galactic disks [28]. The weak-field GR thus can be used to describe the observational features of galactic halos [5,29], of groups and clusters of galaxies [7].

\section{Ricci curvature}

The Lagrangian for $N$-body system interacting by the $\Lambda$ potential [from Eq. (2)] is

$$
\begin{aligned}
L(r, v) & =\frac{1}{2} \sum_{a=1}^{N} m_{a} v_{a}^{2}-U(r), \\
U(r) & =-\sum_{a=1}^{N} \sum_{b=1}^{a-1} \frac{G m_{a} m_{b}}{\left|r_{a}-r_{b}\right|}-\frac{\Lambda}{6} \sum_{a=1}^{N} m_{a}\left|r_{a}\right|^{2} .
\end{aligned}
$$

According to the criterion of relative instability defined in $[23],{ }^{1}$ among two systems the more unstable is the one with smaller negative Ricci curvature

$\mathfrak{r}=\frac{1}{3 N} \inf _{0 \leq s \leq s_{*}} \mathfrak{r}_{u}(s), \quad \mathfrak{r}<0$,

within $0 \leq s \leq s_{*}$ interval of geodesic in the configurational space.

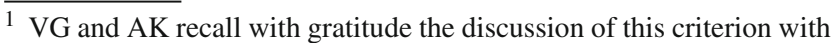
Vladimir Arnold and his important comments, as mentioned in [23].
This criterion follows from the equation of geodesic deviation (Jacobi-Levi-Civita) equation [18,19] averaged via the deviation vector

$\frac{\mathrm{d}^{2} z}{\mathrm{~d} s^{2}}=-\frac{1}{3 N} \mathfrak{r}_{u}(s)+\left\langle\left\|\nabla_{u} n\right\|^{2}\right\rangle$,

where

$n=z \hat{n}, \quad\|\hat{n}\|^{2}=1$,

and $\mathfrak{r}_{u}(s)$ is the Ricci curvature in the direction of the velocity of the geodesic $u$, and

$\mathfrak{r}_{u}(s)=\frac{\operatorname{Ric}(u, u)}{u^{2}}=\sum_{\mu=1}^{3 N-1} K_{\mathbf{e}_{\mu}, u}(s), \quad\left(\mathbf{e}_{\mu} \perp u, \mathbf{e}_{\mu} \perp \mathbf{e}_{v}, \mu \neq v\right)$.

The Ricci tensor for $N$-body system yields [20,23]

$$
\begin{aligned}
\operatorname{Ric}_{\alpha \beta}=- & \frac{1}{2} \frac{\Delta W}{W} g_{\alpha \beta}-\frac{(3 N-2)}{2} \frac{W_{\alpha \beta}}{W} \\
& +\frac{3(3 N-2)}{4} \frac{W_{\alpha} W_{\beta}}{W^{2}}-\frac{(3 N-4)}{4} \frac{\|\mathrm{d} W\|^{2}}{W^{2}} g_{\alpha \beta},
\end{aligned}
$$

where $g_{\alpha \beta}=m_{a} \delta_{\alpha \beta}$, and

$$
\begin{aligned}
W & =E-U=E+\sum_{a=1}^{N} \sum_{b=1}^{a-1} \frac{G m_{a} m_{b}}{\left|r_{a}-r_{b}\right|}+\frac{\Lambda}{6} \sum_{a=1}^{N} m_{a}\left|r_{a}\right|^{2} \\
& =\frac{1}{2} \sum_{a=1}^{N} m_{a} v_{a}^{2} .
\end{aligned}
$$

For the Lagrangian of Eq. (3) the latter is

$$
\begin{aligned}
\operatorname{Ric}(v, v)= & \frac{(3 N-2)}{2 W} \sum_{c=1}^{N} \sum_{\substack{a=1 \\
a \neq c}}^{N} \frac{G m_{c} m_{a}}{\rho_{c a}^{3}} \\
& \times\left(v_{c} \cdot v_{c a}-3 \frac{\left(r_{c a} \cdot v_{c}\right)\left(r_{c a} \cdot v_{c a}\right)}{\rho_{c a}^{2}}\right) \\
+ & \frac{3(3 N-2)}{4 W^{2}}\left(-\sum_{c=1}^{N} \sum_{\substack{a=1 \\
a \neq c}}^{N} G m_{c} m_{a} \frac{r_{c a} \cdot v_{c}}{\rho_{c a}^{3}}\right. \\
& \left.+\frac{\Lambda}{3} \sum_{c=1}^{N} m_{c} r_{c} \cdot v_{c}\right)^{2} \\
- & \frac{(3 N-4)}{2 W} \sum_{c=1}^{N} m_{c} \mid-\sum_{\substack{a=1 \\
a \neq c}}^{N} G m_{a} \frac{r_{c a}}{\rho_{c a}^{3}}+\frac{\Lambda}{3} r_{c} \\
& -\frac{2(3 N-1)}{3} \Lambda,
\end{aligned}
$$


where $r_{a b}=r_{a}-r_{b}, v_{a}=\dot{r}_{a}, v_{a b}=v_{a}-v_{b}, \rho_{a}=\left|r_{a}\right|$, $\rho_{a b}=\left|r_{a b}\right|$, for any two vectors $\mathbf{e}_{1}$ and $\mathbf{e}_{2}$ we have $\mathbf{e}_{1} \cdot \mathbf{e}_{2}=$ $\delta_{i j} e_{1}^{i} e_{2}^{j}$.

\section{Results}

We simulated the dynamics of two types of $N$-body spherical systems of typical galaxy cluster parameters, one defined by Newtonian gravity, the other defined by an additional $\Lambda$ potential i.e. by the Lagrangian of Eq. (3). The Ricci curvature was estimated for both, to see if the instability properties of the two systems according to the criterion of Eq. (5) do reveal differences during their evolution over cosmological time scale.

To simulate systems with typical parameters of galaxy clusters we used a spherical distribution of $N=1,000$ particles (galaxies), each of mass $m=10^{11} M_{\odot}$, inside a sphere of $R=1.5 \mathrm{Mpc}$. The velocities were defined by considering such galaxy clusters (i.e. of 1,000 members) as semivirialized configurations, i.e.

$\sigma^{2}=\frac{G N m}{R}=\frac{G M}{R}$

where $\sigma^{2}$ is the velocity dispersion of galaxies of the cluster and $M$ is the total mass of the cluster. Consequently, the dynamical time scale for a typical cluster in Newtonian and $\Lambda$-modified regime will be

$$
\begin{aligned}
t_{G} & =\left(\frac{2 R^{3}}{G M}\right)^{1 / 2}=3.86 \mathrm{Gyr}, \\
t_{G \Lambda} & =\left(\frac{2 R}{\frac{G M}{R^{2}}-\frac{\Lambda R}{3}}\right)^{1 / 2}=3.90 \mathrm{Gyr} .
\end{aligned}
$$

The results of the computations using Eqs. (5)-(10) are shown in Fig. 1. Comparing Newtonian and $\Lambda$-modified gravity it turns out that the behaviors of the Ricci curvature $\mathfrak{r}$ for the two cases are similar (Fig. 1). However, while the values of the Ricci curvatures practically coincide at time scales up to around $2 \mathrm{Gyrs}$, at later phases those values for the pure Newtonian case are systematically larger than for $\Lambda$-modified gravity. According to the criterion of Eq. (5) in view of the fact that the value $\mathfrak{r}_{G \Lambda}$ is smaller than $\mathfrak{r}_{G}$ - both having negative infimum according to the criterion of Eq. (5) within the cosmological time interval [2.4 Gyr-17.4 Gyr] one can conclude that spherical Newtonian systems, which in the large $N$ limit are known to be exponentially unstable (chaotic) [20], become even more unstable with the $\Lambda$-term in the gravity force of Eq. (2).

Then we performed the same analysis for systems of parameters of superclusters, using the data of the Virgo Supercluster. Note that there is a principal difference between this case and those of galaxy clusters for $\Lambda$-modified gravity

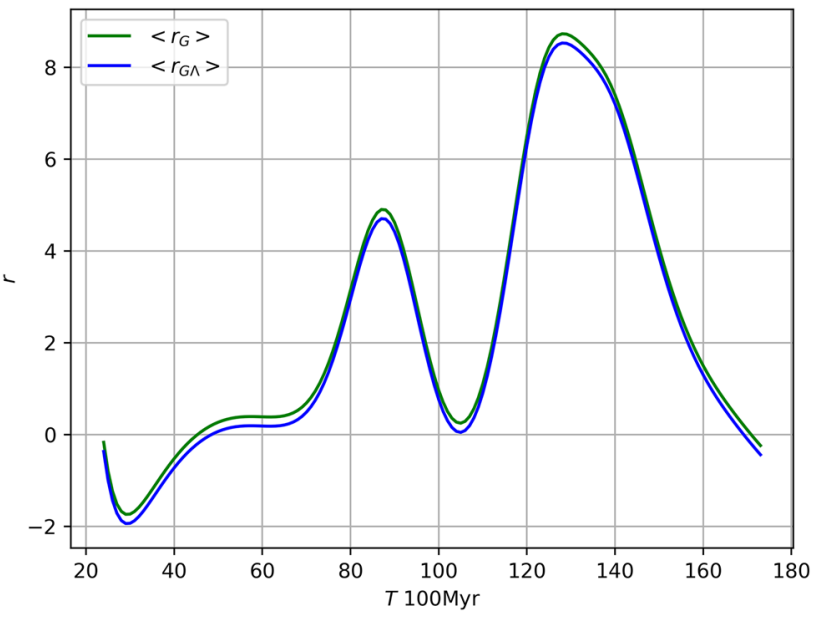

Fig. 1 The Ricci curvature variation vs cosmological time for galaxy cluster parameters in Newtonian (green) and $\Lambda$-modified gravity (blue) regimes

(Fig. 2). Namely, from Eqs. (1) and (2) one can define a critical distance scale for a system, where the repulsive term of $\Lambda$ becomes dominant [8] over the Newtonian gravity

$r_{\text {crit }}^{3}=\frac{3 G M}{\Lambda}$.

For structures smaller than superclusters' scale this radius lies outside the configuration, which means that the role of the $\Lambda$ term in its properties is suppressed. But for superclusters of scales larger than $r_{\text {crit }}$ the role of the $\Lambda$-term can be felt in the dynamics of galaxies [8]. For Virgo Supercluster that critical radius is around $12.66 \mathrm{Mpc}$. In this regard, we checked the behavior of the Ricci curvature for three different cases. First, we analyzed a system of parameters of the Virgo Supercluster, i.e. $R=16.5 \mathrm{Mpc}, N=1,480$, and $M=1.48 \times 10^{15} M_{\odot}$. The results, i.e. the difference of the Ricci curvature for Newtonian and $\Lambda$-modified gravity, are given in Fig. 3. Then we studied two different cases, i.e. with the same mass and number of particles but for different radii, i.e. $R=18(>12.66) \mathrm{Mpc}$ and $R=10 \mathrm{Mpc}(<12.66)$. For these two cases the results are shown in Figs. 4 and 5. It is interesting that for both, $18 \mathrm{Mpc}$ and $16.5 \mathrm{Mpc}$ (both exceeding the critical distance $12.66 \mathrm{Mpc}$ ), as time goes on the difference of Ricci is increasing and even for $R=18$ $\mathrm{Mpc}$, it becomes positive, which can be interpreted as a tendency to a free particle system. Meanwhile, for the bound structure $R=10 \mathrm{Mpc}$ the Ricci curve shows a tendency to decrease, i.e. indicating the unstable $N$-body system.

\section{Conclusions}

The weak-field GR modified based on the Newton theorem enabled one the common description of the dark matter and 


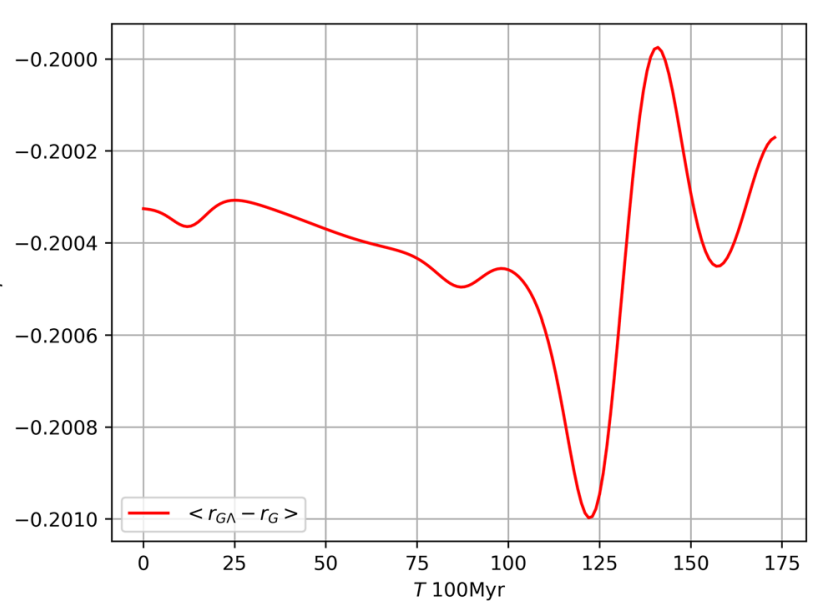

Fig. 2 The difference of the Ricci curvature values of curves in Fig. 1, i.e. at Newtonian and $\Lambda$-modified gravity laws

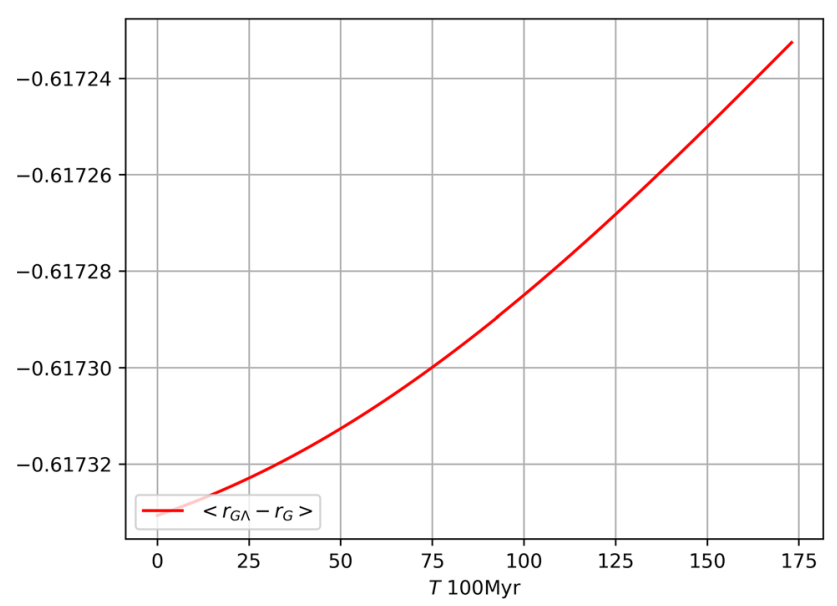

Fig. 3 The same as in Fig. 2 but for supercluster (Virgo) parameters

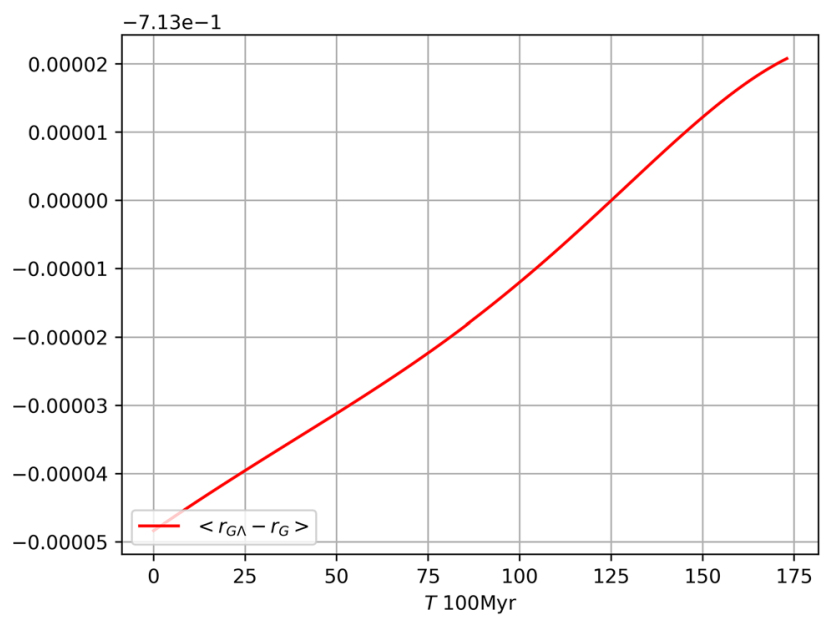

Fig. 4 The same as in Fig. 3 but for $R=18 \mathrm{Mpc}$

dark energy $[5,6]$, as weak-field and cosmological manifestation of GR, respectively, both determined by the cosmological constant.

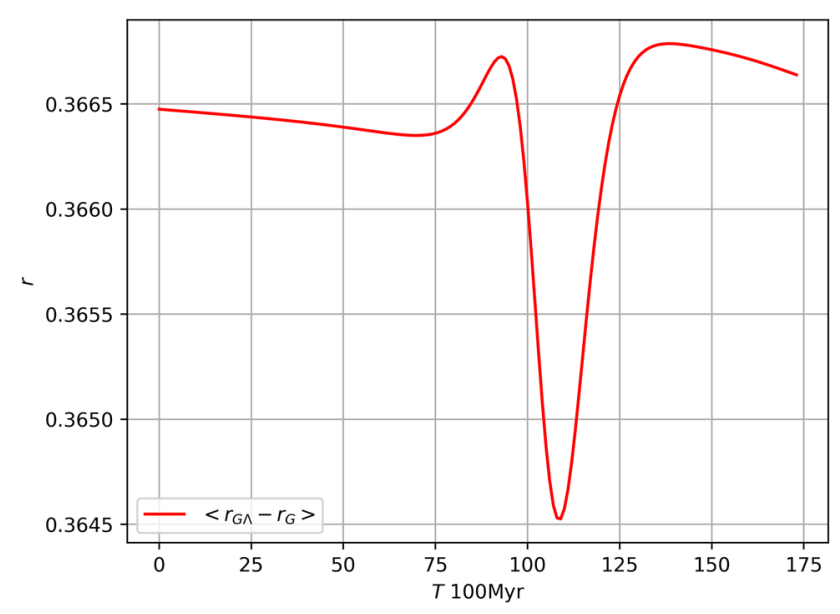

Fig. 5 The same as in Fig. 3 but for $R=10 \mathrm{Mpc}$

As further step to probe that GR modification, we analyzed its possible role in the evolution of the galaxy clusters, i.e. at spatial scales where the repulsive gravity term becomes nonnegligible. We used the Ricci curvature criterion to follow the comparative instability of two type of spherical systems, i.e. those evolving according to modified $\Lambda$-gravity of Eqs. (1) and (2) with respect to usual Newtonian systems.

Our main conclusions can be formulated as follows:

(a) The studied types of systems of galaxy cluster parameters do reveal discrepancy in their instability properties during the evolution, namely, the $\Lambda$-modified gravity systems tend to become more unstable with respect to those described by Newtonian law. The discrepancy starts to be visible at cosmological times i.e. at time scales exceeding roughly $2 \mathrm{Gyr}$.

(b) The supercluster (Virgo) parameter systems reveal differences in their instability properties depending on their spatial scales. Namely, at distance scales where the $\Lambda$-term dominates over the Newtonian gravity, the systems tend to free particle systems at cosmological time scales, while at smaller distances their behavior remains unstable as of the galaxy clusters, as expected.

We note that for $f(R)$-gravity, "beyond Horndeski" covariant Galileon models have been already used to describe the observable features of clusters of galaxies [30-32], thus indicating the suitability of the latter for testing of modified gravity theories.

The study of the evolutionary effects of galaxy clusters at dedicated numerical simulations (including using advanced methods of the theory of dynamical systems [33]) can provide additional tests to $\Lambda$-gravity as the weak-field limit for General Relativity.

Acknowledgements AS is partially supported by the ICTP through AF-04. 
Data Availability Statement This manuscript has no associated data or the data will not be deposited. [Authors' comment: All data generated or analysed during this study are included in this published article.]

Open Access This article is licensed under a Creative Commons Attribution 4.0 International License, which permits use, sharing, adaptation, distribution and reproduction in any medium or format, as long as you give appropriate credit to the original author(s) and the source, provide a link to the Creative Commons licence, and indicate if changes were made. The images or other third party material in this article are included in the article's Creative Commons licence, unless indicated otherwise in a credit line to the material. If material is not included in the article's Creative Commons licence and your intended use is not permitted by statutory regulation or exceeds the permitted use, you will need to obtain permission directly from the copyright holder. To view a copy of this licence, visit http://creativecomm ons.org/licenses/by/4.0/.

Funded by SCOAP ${ }^{3}$.

\section{References}

1. A.G. Riess et al., ApJ 855, 13 (2018)

2. A.G. Riess et al., ApJ 876, 85 (2019)

3. L. Verde, T. Treu, A.G. Riess. arXiv:1907.10625

4. E. Di Valentino et al. arXiv:1910.09853

5. V.G. Gurzadyan, Eur. Phys. J. Plus 134, 98 (2019)

6. V.G. Gurzadyan, A. Stepanian, Eur. Phys. J. C 78, 632 (2018)

7. V.G. Gurzadyan, A. Stepanian, Eur. Phys. J. C 79, 169 (2019)

8. V.G. Gurzadyan, A. Stepanian, Eur. Phys. J. C 79, 568 (2019)

9. V.G. Gurzadyan, A. Stepanian, Eur. Phys. J. Plus 134, 98 (2019)

10. V.G. Gurzadyan, R. Penrose, Eur. Phys. J. Plus 128, 22 (2013)

11. N.J. Poplawski, Eur. Phys. J. C 79, 734 (2019)
12. S. Kopeikin, M. Efroimsky, G. Kaplan, Relativistic Celestial Mechanics of the Solar System (Wiley, Amsterdam, 2001)

13. M. Eingorn, ApJ 825, 84 (2016)

14. S. Turyshev, Phys. Uspekhi 52, 1 (2009)

15. I. Ciufolini et al., Eur. Phys. J. C 79, 872 (2019)

16. T.E. Collett et al., Science 360, 1342 (2018)

17. V.G. Gurzadyan, A. Stepanian, Eur. Phys. J. C 78, 869 (2018)

18. D.V. Anosov, Geodesic flows on closed Riemannian manifolds of negative curvature. Proc. Steklov Math. Inst. 90, 1 (1967)

19. V.I. Arnold, Mathematical Methods of Classical Mechanics (Springer, New York, 1989)

20. V.G. Gurzadyan, G.K. Savvidy, A\&A 160, 203 (1986)

21. A.A. Kocharyan, Commun. Math. Phys. 143, 27 (1991)

22. A.V. Gurzadyan, A.A. Kocharyan, Int. J. Mod. Phys. D 26, 1741019 (2017)

23. V.G. Gurzadyan, A.A. Kocharyan, Ap. Space Sci. 135, 307 (1985)

24. A.A. El-Zant, V.G. Gurzadyan, Physica D 122, 241 (1998)

25. E. Fermi, J. Pasta, S. Ulam, Los Alamos National Laboratory, LA$1940(1955)$

26. A.E. Allahverdyan, V.G. Gurzadyan, Phys. Rev. E 93, 052125 (2016)

27. V.G. Gurzadyan, Observatory 105, 42 (1985)

28. A.V. Kravtsov, ApJ Lett. 764, L31 (2013)

29. V.G. Gurzadyan et al., A\&A 609, A131 (2018)

30. S. Capozziello, E. De Filippis, V. Salzano, MNRAS 394, 947 (2009)

31. V. Salzano et al., JCAP 10, 033 (2016)

32. S. Capozziello et al., MNRAS 474, 2430 (2018)

33. A.V. Gurzadyan, A.A. Kocharyan, Eur. Phys. J. C 76, 685 (2016) 